\title{
ESTUDO DA DEPRESSÃO CIRCULAR DE COLÔNIA-sp PELO MÉTODO SÍSMICO
}

\author{
FERNANDO A. NEVES
}

\begin{abstract}
Study of the circular depression of Colônia-sp by seismic methods In the region of Colônia, about $35 \mathrm{Km}$ south of the city of São Paulo, occurs an almost circular depression with a diameter of $3.64 \mathrm{Km}$, within the pre-cambrian crystalline basement and filled with Terciary and Quaternary sediments. Based on previous geological and geophysical data, the structure is interpreted as the result of a meteorite impact. The depth of sediment/basement interface was estimated from six seismic lines. Results from one-dimensional forward modelling and seismic interpretation of field records, show that the maximum depth of the interface lies between 380 and 450 meters. The formation of the depression is interpreted to be younger than 36.3 million of years.
\end{abstract}

Keywords: Seismic, Astrobleme, Meteor Impact, São Paulo, Brasil.

RESUMO A aproximadamente $35 \mathrm{Km}$ ao sul da cidade de São Paulo, na região de Colônia, existe uma depressão quase circular com diâmetro de $3,64 \mathrm{Km}$, situada no embasamento cristalino pré-cambriano e preenchida com sedimentos terciários e quaternários. Dados geológicos e geofísicos sugerem que a estrutura originou-se por impacto meteorítico. Para estimar a profundidade do embasamento foram realizadas seis linhas sísmicas. Ós resultados obtidos a partir do modelamento direto unidimensional e da interpretação dos registros sísmicos mostram que a profundidade máxima da interface entre sedimentos e embasamento situa-se entre 380 e $450 \mathrm{~m}$. A formação da depressão é admitida ser mais recente do que 36.3 milhões de anos.

Palavras-chave: Sísmica, Astroblema, Impacto Meteorítico, São Paulo, Brasil.

INTRODUÇÃO Fotografias aéreas e mapas topográficos da região de Colônia-SP (Fig. 1) mostram uma clara depressão quase circular. A estrutura consiste de uma parte central plana aluvial pantanosa (Riccomini et al. 1991), com diâmetro de aproximadamente 3,64 km, circundada por morros dispostos em anel, cujas alturas atingem até $125 \mathrm{~m}$ acima da planície.

A origem desta feição é ainda incerta. Investigações geológicas complementadas com dados geofísicos gravimétricos, de resistividade e magnetotelúricos, foram realizadas com intuito de investigar a origem desta depressão.

Baseando-se em argumentos morfológicos, Kollert et al. (1961) propõem que a depressão de Colônia se originou por dissolução de rochas calcárias ou por impacto meteorítico. Posteriormente, Crosta (1982), também em argumentos morfológicos, reforçou a hipótese de estrutura de impacto. Riccomini et al. (1991) rejeitam a hipótese de dissolução de rochas calcárias, em virtude deste tipo rocha jamais ter sido registrado na região.

Outras hipóteses, relacionadas a um padrão construtivo de interferência estrutural e intrusões e explosões vulcânicas são também rejeitadas, devido à existência de um padrão estrutural ENE-WNW bem definido, e à inexistência de rochas plutônicas e vulcânicas na área. Assim, resta apenas a hipótese de impacto meteorítico como gerador da depressão (Riccomini et al 1991).

Existem, presentemente, na face da Terra cerca de 150 estruturas de impacto (Grieve \& Robertson 1979), ou astroblemas(Dietz 1961). As estruturas de impacto estão divididas em três categorias (Grieve \& Robertson 1979):

(1) Comprovadas: quando pedaços ou fragmentos c!o meteorito são encontrados;

(2) Prováveis: quando há registro de estruturas dos efeitos do impacto, principalmente o metamorfismo de choque sobre a rocha alvo, tais como, cones de estilhaçamento (shatter cones) e minerais formados em altas pressões (coesita e estishovita);

(3) Possíveis: quando existem feições morfológicas típicas como depressões de formato aproximadamente circular, rá ão diâmetro/profundidade da cratera compatíveis, mas onde o metamorfismo de choque não está caracterizado.
Segundo Crosta (1982), no Brasil existem cerca de seis estruturas de impacto. A depressão de Colônia é classificada como uma possível estrutura desta natureza, devido à inexistência de evidências diretas, tais como fragmentos do meteorito ou estruturas e feições de metamorfismo de choque.

As investigacões geofísicas (levantamentos gravimétricos e de eletroresistividade) de Kollert et al. (1961) estimaram entre 285 e $400 \mathrm{~m}$, a profundidade da interface sedimento/embasamento. As investigações gravimétricas de Motta \& Flexor (1991), estimaram entre $300-350 \mathrm{~m}$ a profundidade da cratera, e as magnetotelúricas de Masero \& Fontes (1992) entre 200 e $355 \mathrm{~m}$, mas com profundidade máxima até o topo do embasamento de $500 \mathrm{~m}$.

O objetivo deste artigo é melhorar a estimativa da profundidade do contato sedimento/embasamento na depressão de Colônia. Com esta finalidade, seis linhas sísmicas foram levantadas no interior da cratera.

GEOLOGIA A estrutura de Colônia situa-se em rochas do embasamento pré-cambriano do Cinturão Dobrado Ribeira (Almeida 1964), o qual consiste de rochas metamórficas do Ciclo Transamazônico, remobilizadas durante o Ciclo Brasiliano. O Cinturão Dobrado Ribeira é interpretado como uma zona de convergência litosférica ocorrida durante o Ciclo Brasiliano. Os principais litotipos presentes na região compreendem gnaisses, migmatitos, dioritos, micaxistos, filhos, quartzitos, milonitos, granitos e granodioritos (Sadowski 1974, Coutinho 1980). A sudeste da cratera predominam gnaisses e, a noroeste, intrusões graníticas (Kollert et al. 1961). Também estão presentes na região, sedimentos cenozóicos de idade oligocênica, correlates aos das bacias trafogênicas de São Paulo, Taubaté, Resende e Volta Redonda (Riccomini et al 1987).

A estrutura regional do embasamento é caracterizada por blocos limitados por falhas, possivelmente muito profundas, de direção ENE-WNW (Fig. 1). Rochas sedimentares afloram na borda sul e sudeste da estrutura. São arenitos correlates aos da Formação Resende (Riccomini et al 1987). O interior da depressão é preenchido por depósitos quaternários ricos em arguas e componentes orgânicos, tratando-se de uma região de pântano (Riccomini et al 1992). 


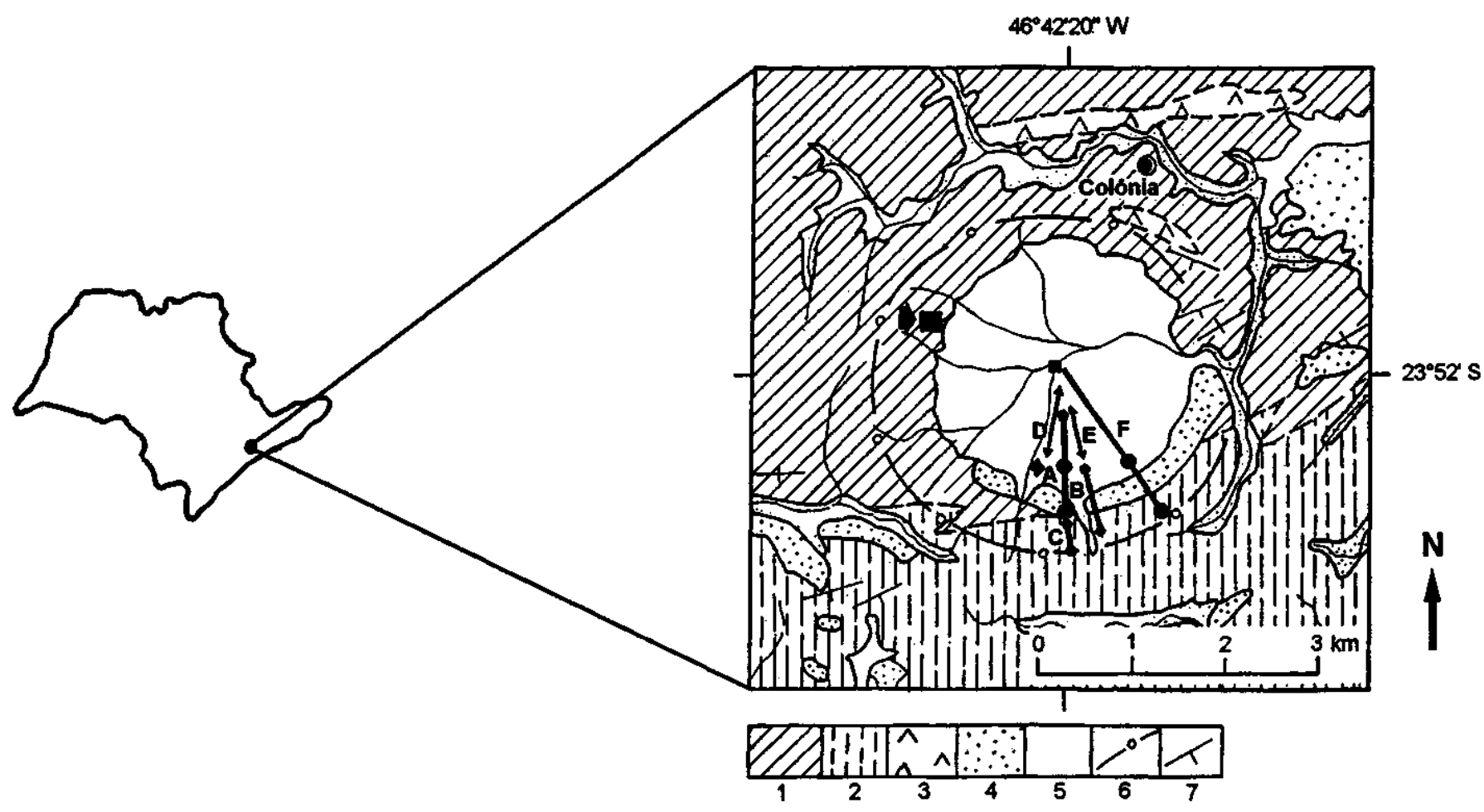

Figura 1 Localização da depressão circular de Colônia. Em detalhe é o Mapa geológico da área (Continha 1980, modificado porRiccomini et al. 1991). 1 micaxistos, quartzitos; 2. gnaisses, migmatitos, milonitos; 3. dioritos e quartzo- dioritos; 4 . sedimentos terciários; 5. depósitos aluviais quaternários; 6. anel circular externo; 7. orientação geral das estruturas do embasamento cristalino. Localização das seis linhas sísmicas levantadas. ${ }^{\bullet}$ pontos de tiro para afoute explosiva (linhas A, B, C e F), $\mathbf{\Delta}$ fonte com o martelo (linhas $D$ e E). $\bullet$ centro geométrico da depressão, $=>$ Colônia Penal; $\square$ localização do poço. Figure 1. Location of the circular depression of Colônia. The inset is the geological map of the área (Coutinho 1980, modified by Riccomini et al. 1991). 1. mica schists, quartzites; 2. gneisses, migmatites, mylonites; 3 . diorites and quartz-diorites; 4. tertiary sediments; 5 . quaternary alluvial deposits; 6 . crater rim; 7. structural trend of the crystalline basement. Location of the $\dot{u}$ । seismic lines. $\bullet$ shot points forexplosive source (lines A, B, C and F); A sources with hammer (lines D and E); geometrical center of the depression, $\square$ presidium; $\square$ location ofa well.

A idade da formação da depressão, se devido a impacto meteorítico, é baseada em parâmetros de preservação morfológica de crateras de impacto. Grieve \& Robcrtson (1979) fornecem uma relação empírica que estabelece uma relação entre a idade, diâmetro e grau de preservação do astroblema (por exemplo, grau de erosão das colinas que circundam a cratera). Usando-se esta relação, estima-se uma idade máxima para a estrutura de Colônia entre 36 (Eoceno-Oligoceno) e 5 (Mioceno-Plioceno) milhões de ano. A existência de rochas sedimentares correlatas à Formação Resende na área da cratera, está de acordo com a estimativa de 36 m.a. para a idade máxima de formação da depressão.

MÉTODO SÍSMICO O método sísmico permite a determinação da distribuição espacial das camadas em subsuperfície através do espalhamento de ondas sísmicas geradas por fontes sísmicas controladas. Além da geometria das camadas, o método sísmico também permite a extração de parâmetros petrofísicos destas, tais como velocidade de propagação e densidade das rochas. $\mathrm{O}$ conhecimento da velocidade de propagação das ondas sísmicas permite construir a seção da área pesquisada em profundidade, e estimar a profundidade da depressão de Colônia com maior acuracidade. Isto porque, o método sísmico possui maior resolução espacial que os métodos potenciais (gravimétricos, magnetotelúricos, etc.). Somente as ondas longitudinais (ondas P) foram analisadas neste trabalho.

Os levantamentos foram concentrados na borda sul da depressão devido as dificuldades operacionais (permissão para acesso e detonação) ocorridas na borda norte, onde existe uma Colônia Penal. Além disso, não foi possível alcançar o centro geométrico da cratera, devido ao pântano da região central.

A posição das seis linhas sísmicas levantadas é mostrada na figura 1. Em quatro delas (A, B, C e F), a fonte sísmica foi a dinamite em carga moldada (círculos, Fig. 1). Nas demais (D e E), a fonte sísmica foi o martelo (triângulos, Fig. 1). As linhas levantadas com fonte explosiva visaram definir interfaces mais profundas, e as demais a velocidade das camadas superficiais.

As cargas foram detonadas num intervalo de profundidade de 1-2 $\mathrm{m}$ a partir da superfície, usando-se espoletas sismográficas. $\mathrm{O}$ uso da carga moldada visou direcionar a energia verticalmente para baixo.

O espaçamento entre receptores foi de 4 ou $10 \mathrm{~m}$ nas linhas com martelo e de 30, 45 ou $90 \mathrm{~m}$ nos perfis com fonte explosiva. Os receptores eram geofones de relutância variável (bobina móvel) de frequência natural de $28 \mathrm{~Hz}$. Os dados sísmicos foram registrados com o registrador digital TEAC de 14 bits, de 8 canais. Um dos canais foi alocado para o tempo-zero de registro (trigger), enquanto 7 canais destinaram-se para o registro multiplexado dos dados. Os dados foram armazenados em disquetes de 3.5 pol.. Foram usados alguns módulos de interface com filtros passa-baixa (0-200 $\mathrm{Hz}$ ) do tipo Butterworth de $12 \mathrm{~dB} /$ oitava e amplificadores de $8 \mathrm{~dB}$. O desenho deste filtro visou atenuar ruídos eletrônicos e culturais de alta frequência, tais como, movimentação de pessoas e de máquinas próximo aos receptores, comuns na 


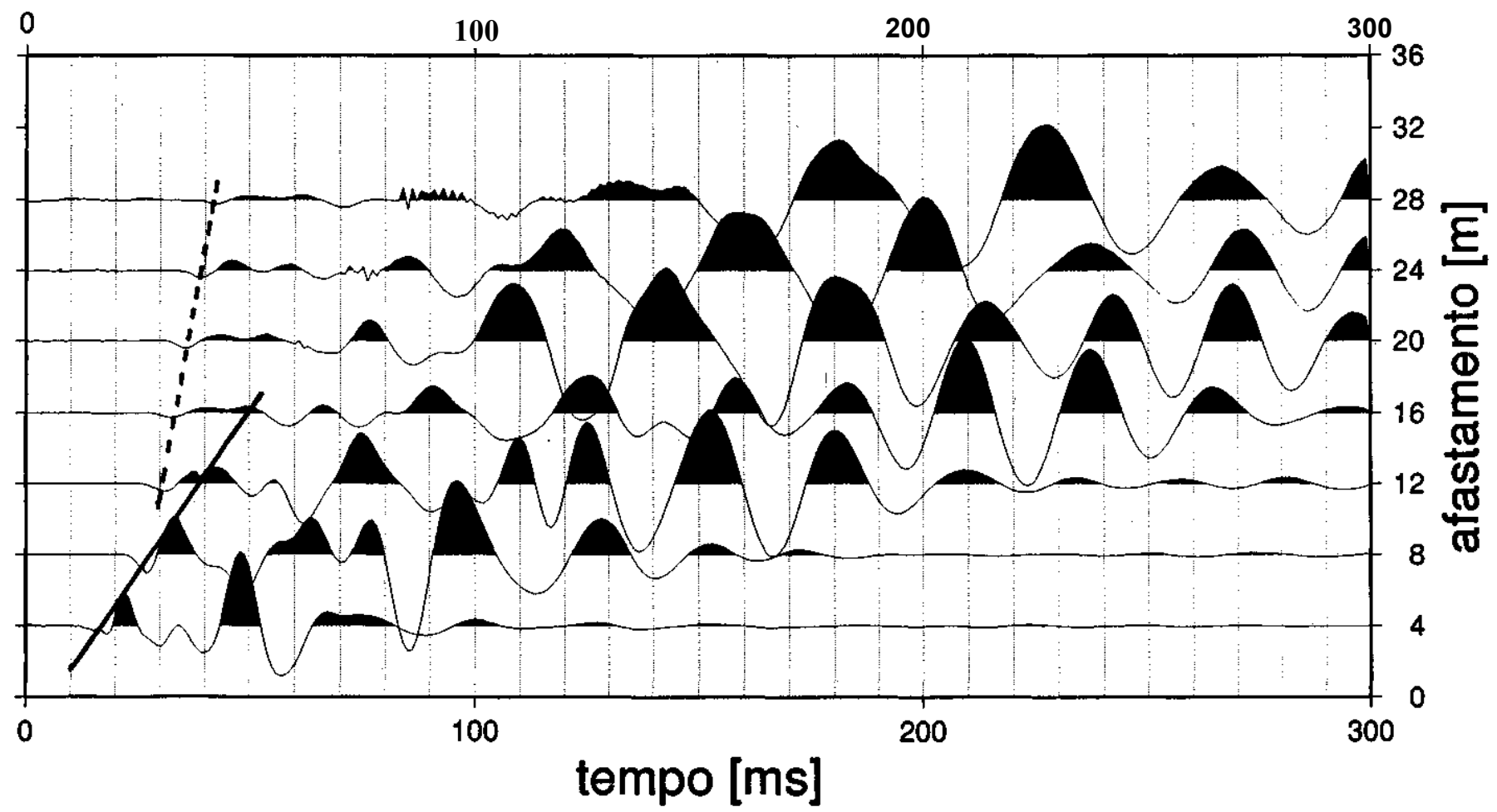

Figura 2. Registro de campo não-processado obtido com o uso do martelo. Linha reta contínua = onda direta $(400 \mathrm{~m} / \mathrm{s}) \mathrm{na}$ camada mais superficial; linha tracejada = a onda refratada $(1400 \mathrm{~m} / \mathrm{s})$ na camada subjacente. As chegadas de grande amplitude são as ondas superficiais.

Figure 2. Raw field data obtained by using hammcr. Straight continuous line $=$ direct wave $(400 \mathrm{~m} / \mathrm{s})$ which propagates through the topmost layer; dashed line $=$ refracted wave $(1400 \mathrm{~m} / \mathrm{s})$ which propagates through the underlying layer. The large amplitude arrivals are surface waves.

região do levantamento. Nenhum filtro anti-alias ou pré-filtro A/D corta-baixa foi utilizado.

Os parâmetros de aquisição usados nas linhas com fonte explosiva foram escolhidos de modo a registrar, preferencialmente, ondas refratadas. Entretanto, as ondas refletidas também puderam ser identificadas nos sismogramas. Excetuando a linha $F$, nas demais foi executado tiro reverso para obter o ângulo de mergulho e estimar corretamente a velocidade da camada mergulhante. Para uma melhor identificação das ondas refratadas nos registros de campo, o arranjo preferencial dos perfis foi do tipo end-on, onde a fonte está situada em umas das extremidades da linha sísmica. A cobertura nominal foi de $100 \%$, i.e., cada ponto em profundidade foi amostrado apenas uma vez.

\section{Processamento e Interpretação dos Dados Os} dados sísmicos foram processados na estação de trabalho SUN Sparc II. As seguintes etapas foram aplicadas aos dados coletados :

- Demultiplexação

- Correção dos efeitos da divergência esférica

- Correções estáticas

- Correção de sobretempo normal (NMO)

- Filtragens digitais

- Ganhos do tipo $A G C$ (controle de ganho automático)

A correção de $N M O$ foi aplicada somente nos sismogramas onde as reflexões eram claramente identificáveis, permitindose um estiramento do traço sísmico de até $15 \%$. Nenhum silenciamento (mutè) foi aplicado nos traços. Não se observou nenhuma significativa anomalia estática foi observada ou associada à variação lateral de velocidade nas camadas superficiais.

As figuras 2 e 3, mostram dois registros de campo não processados, sendo um obtido com martelo e, outro, com fonte explosiva, respectivamente. No sismograma da figura 2, observa-se a predominância das ondas superficiais, principalmente ground-roll. Os eventos de interesse aparecem na janela temporal de 10-100 ms, correspondente a onda direta e a onda refratada. Na figura 3 não se observa a predominância de ondas superficiais. Isto é provavelmente devido ao maior afastamento mínimo (entre fonte e primeiro geofone) e ao tipo de fonte, pois a detonação da dinamite gera uma banda maior de frequência do que a pancada do martelo, e com uma frequência dominante mais alta. No sismograma desta figura, a região de interesse está na janela temporal de $50-250 \mathrm{~ms}$, onde se observa a onda direta e a onda refratada.

A linha E foi levantada sobre um solo pouco consolidado. Gandolfo (1991) identificou neste perfil eventos relacionados a duas camadas. A mais superficial, com espessura de $3 \mathrm{~m}$ e velocidade de $500 \mathrm{~m} / \mathrm{s}$ e a mais profunda com velocidade de $1600 \mathrm{~m} / \mathrm{s}$.

Os perfis A, B, C e F, mostrados nas figuras. 4, 5, 6 e 7, respectivamente, forneceram importantes informações da subsuperfície da área estudada. Apesar da baixa cobertura $(100 \%)$, os sismogramas processados permitem interpretar a existência de dois refratores distintos (Figs. 4 e 7), que se correlacionam às interfaces entre os sedimentos quaternários e terciários, e entre os sedimentos terciários e o embasamento.

A distribuição de velocidade (Fig. 8) interpretada a partir dos sismogramas foi usada num programa de modelamento cinemático direto unidimensional, isto é, com a velocidade variando apenas em profundidade, (Cohen \& Stockwell Jr. 1996). Este programa calcula os tempos de trânsito (tempos 


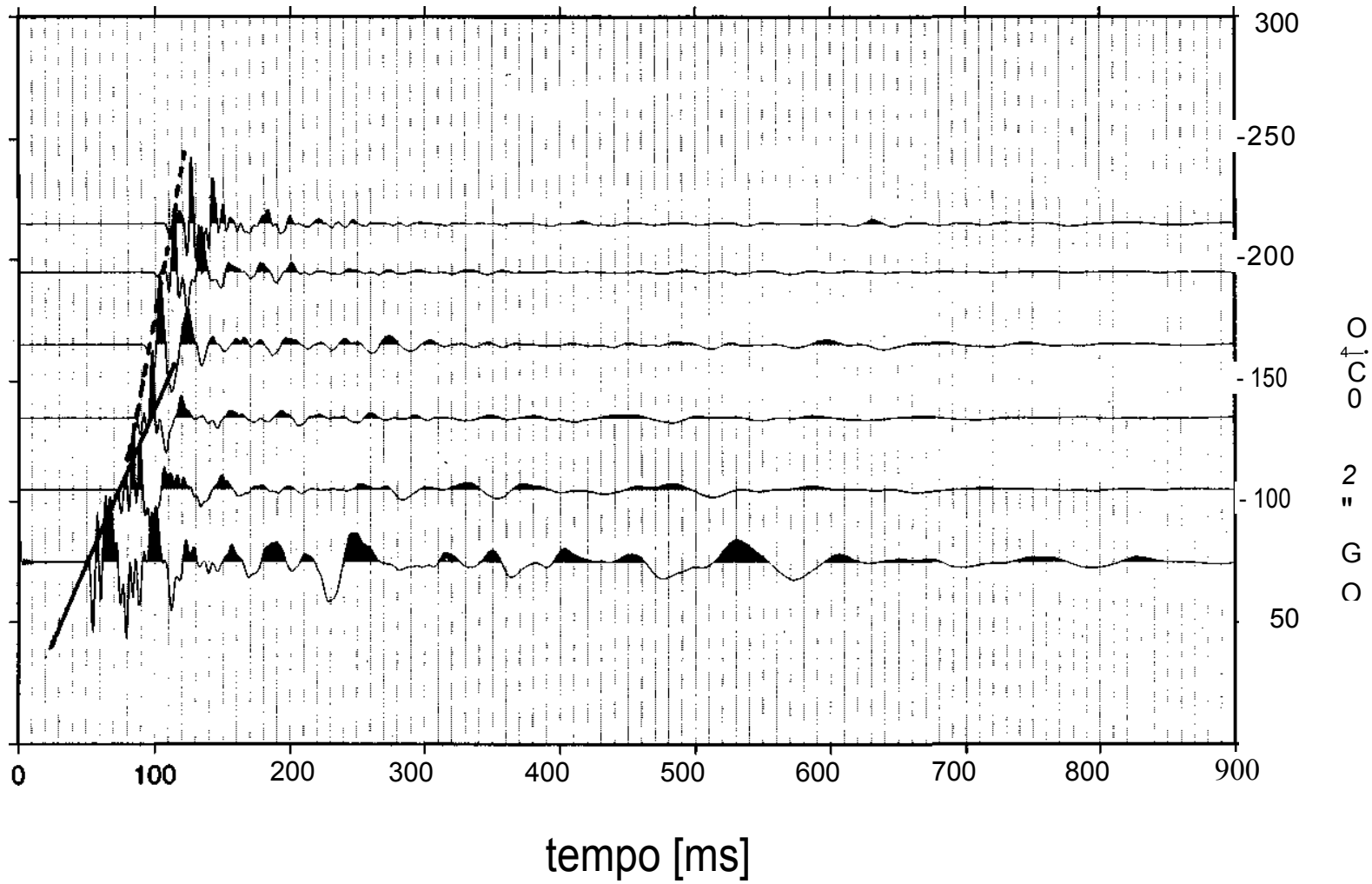

Figura 3. Registro de campo não-processado obtido com o uso de dinamite. Linha reta contínua $=$ a onda direta $(1400 \mathrm{~m} / \mathrm{s})$ propagante na camada mais superficial, linha tracejada = onda refratada $(2200 \mathrm{~m} / \mathrm{s})$ na camada subjacente.

Figure 3. Raw field data obtained by using dynamite. Straight continuous line = direct wave $(1400 \mathrm{~m} / \mathrm{s})$ which propagates through the topmost layer, dashed line $=$ refracted wave $(2200 \mathrm{~m} / \mathrm{s})$ which propagates through the underlying layer.

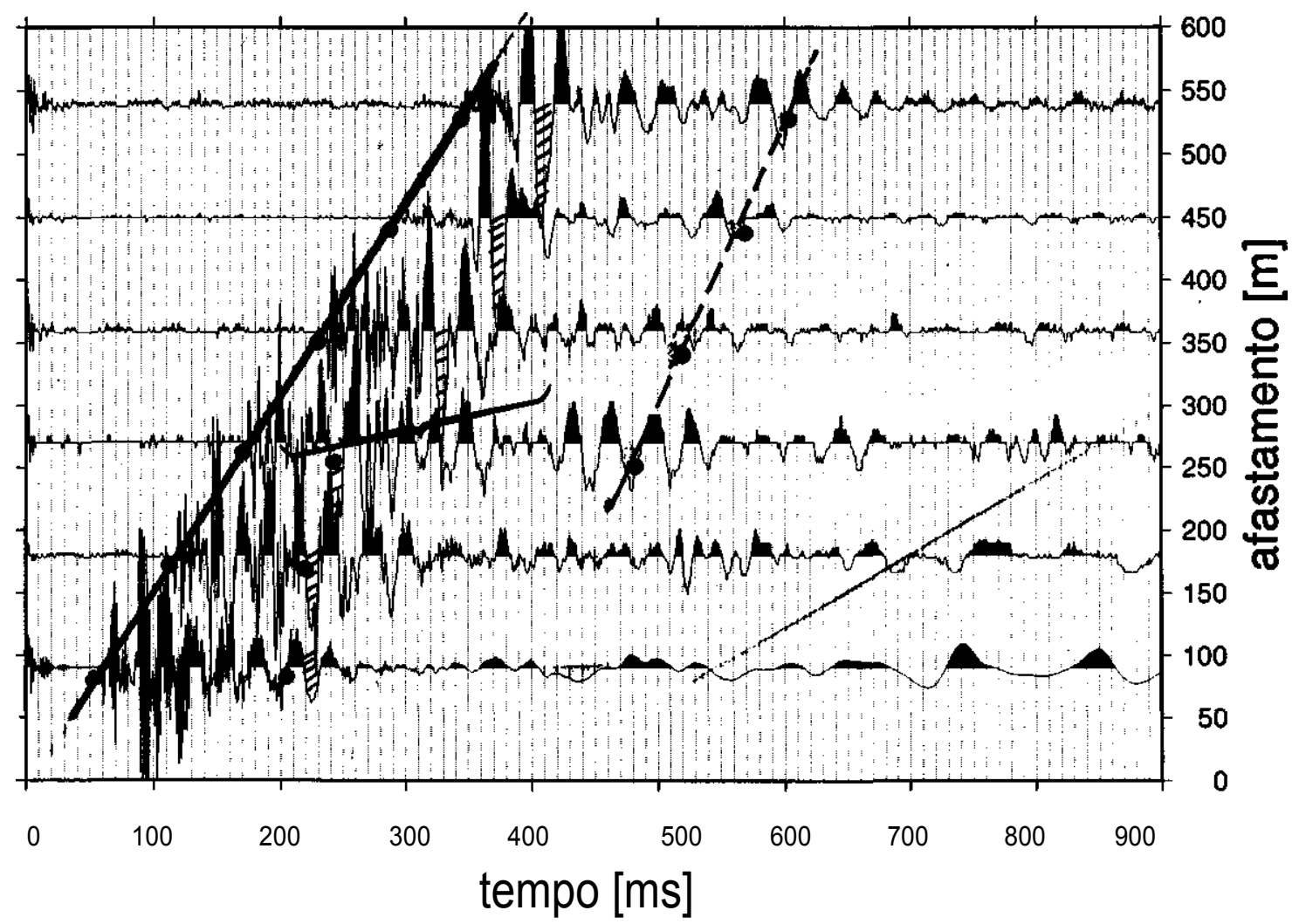

Figura 4. Linha A levantada com dinamite. Linha reta contínua = a onda direta $(1450 \mathrm{~m} / \mathrm{s})$, linha tracejada = segunda onda refratada $(1600 \mathrm{~m} / \mathrm{s})$, com picos hachurados = primeira onda refratada $(2150 \mathrm{~m} / \mathrm{s})$. A localização de uma (possível) falha está indicada com um colchete.

Figure 4. Line A acquired by using dynamite. Straight continuous line $=$ direct wave $(1450 \mathrm{~m} / \mathrm{s})$, dashed line $=$ second refracted wave $(1600 \mathrm{~m} / \mathrm{s}$, with hashed peaks

$=$ first refracted wave $(2150 \mathrm{~m} / \mathrm{s})$. The location of a (possible) fault is shown by a bracket. 


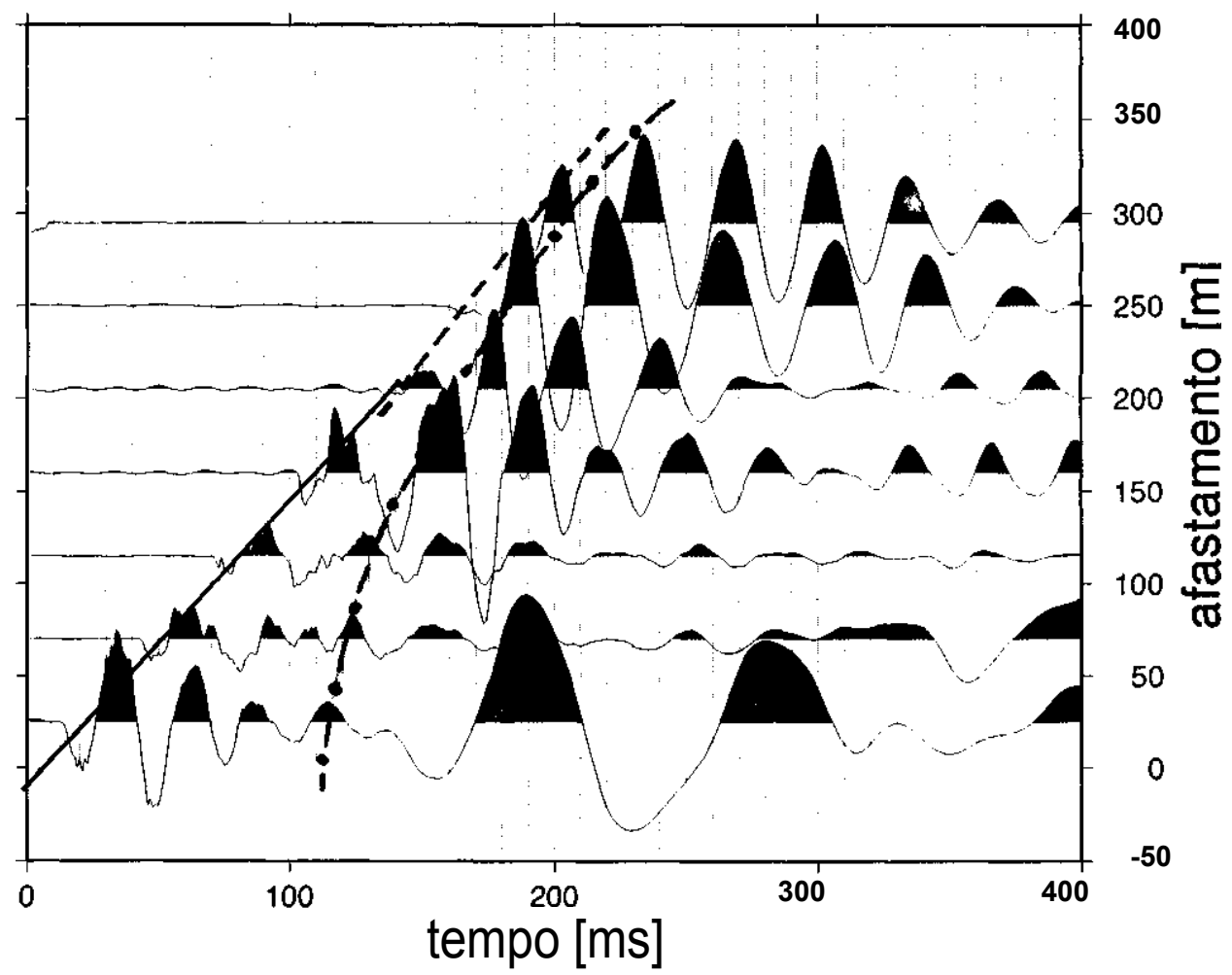

Figura 5. Linha B levantada com dinamite. Linha reta contínua $=$ onda direta $(1400 \mathrm{~m} / \mathrm{s})$, curva com pontos e traços $=$ onda refletida $(1550 \mathrm{~m} / \mathrm{s})$, linha tracejada $=$ onda refratada $(1600 \mathrm{~m} / \mathrm{s})$.

Figure 5. Line B acquired by using dynamile. Straiglit continuoiis Une $=$ clireci wave $(1400 \mathrm{~m} / \mathrm{s})$, dotted-dashed Une $=$ reflected wave $(1550 \mathrm{~m} / \mathrm{s})$, dashed Une $=$ refracted wave $(1600 \mathrm{~m} / \mathrm{s})$.

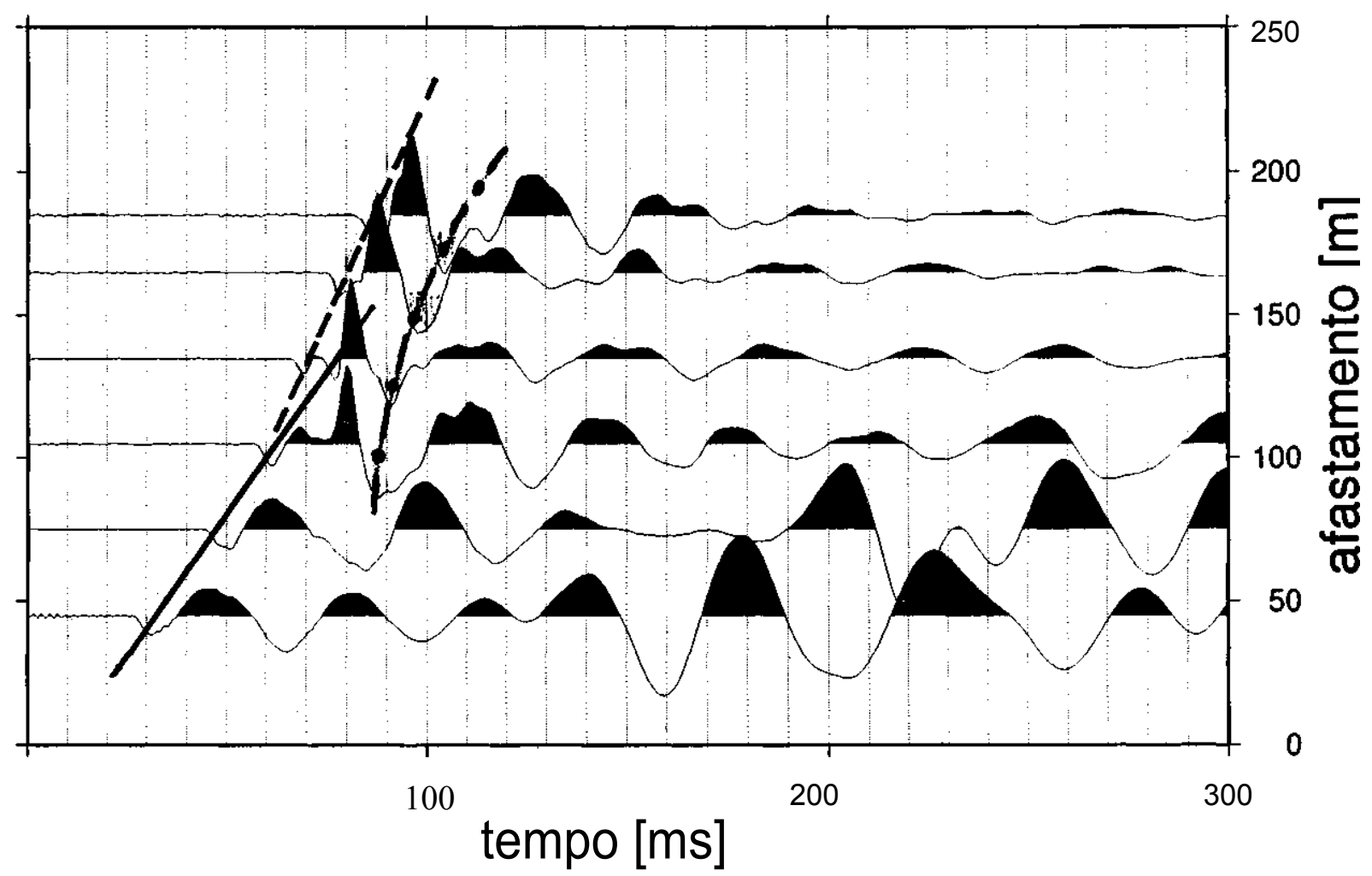

Figura 6. Linha C levantada com dinamite. Linha reta contínua $=$ onda direta $(1450 \mathrm{~m} / \mathrm{s})$, curva com pontos e traços $=$ onda refletida, linha tracejada = onda refratada $(3300 \mathrm{~m} / \mathrm{s})$, esta última interpretada como sendo oriunda do topo do embasamento fraturado.

Figure 6. Line $\mathrm{C}$ acquired by using dynamite. Straight continuous line $=$ direct wave $(1450 \mathrm{~m} / \mathrm{s})$, dotted-dashed Une $=$ reflected wave, dashed line $=$ refracted wave $(3300 \mathrm{~m} / \mathrm{s})$, the latter is interpreted as coming from the top of the fractured basement. 


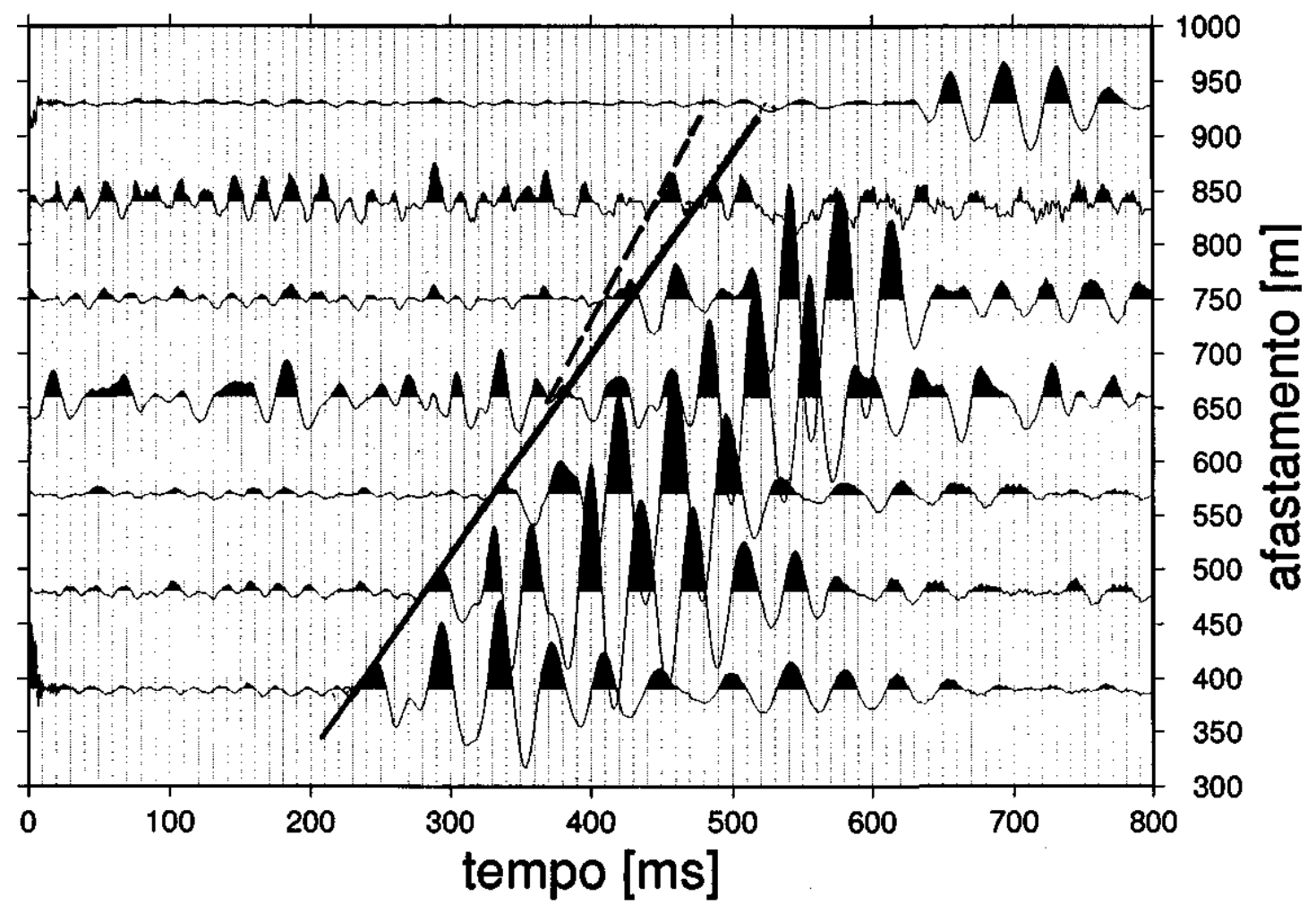

Figura 7. Linha F levantada, com dinamite, no interior da depressão. Linha reta contínua = primeira onda refratada (2150 $\mathrm{m} / \mathrm{s})$, linha tracejada = segunda onda refratada $(3300 \mathrm{~m} / \mathrm{s})$, interpretada como sendo oriunda do topo do embasamento.

Figure 7. Line $\mathrm{F}$ acquired, using dynamite, in the inner part of the depression. Straight continuous line $=$ first refracted wave $(2150 \mathrm{~m} / \mathrm{s})$, dashed line $=$ second refracted wave $(3300 \mathrm{~m} / \mathrm{s})$, the latter is interpreted as coming from the top of the basemcnt.

sintéticos) das ondas P para um modelo com camadas de velocidade constante. Os tempos sintéticos obtidos a partir da distribuição de velocidade da figura 8 estão representados na figura 4 como círculos sólidos. Nota-se que há uma razoável concordância entre os tempos sintéticos e os observados (interpretados). Pequenas discrepâncias podem ser atribuídas a variações laterais de velocidade, erro no sistema de tempozero e imperfeições nas correções estáticas. Esta concordância indica que a distribuição de velocidade obtida a partir da interpretação dos sismogramas é confiável.

Com base na interpretações dos sismogramas e no modelamento direto dos dados sísmicos foi possível construir uma seção na porção centro-sul (Fig. 8) do material que preenche a depressão em profundidade. Esta seção mostra que a morfologia da camada terciária e a da superfície do topo do embasamento são aproximadamente concordantes, sugerindo que à época de formação da depressão, esta camada terciária já existia. Datações palinológicas por Riccomini et al (1991) estimam que é de 36 m.a., a idade dos sedimentos da camada terciária, limite máximo da formação da depressão. A existência desta camada terciária foi confirmada por amostras de testemunho de sondagem da companhia Air Drill (Ronaldo Klockner, comunicação verbal). Fragmentos destes sedimentos e de rochas do embasamento coletados durante esta perfuração foram, petrograficamente, analisados por Jocl Valença (comunicação verbal). Nenhuma evidência de metamorfismo de impacto foi observada nas lâminas daquelas amostras. Contudo, considerando que a energia de impacto de um meteoro decai com a sexta potência da distância a partir do ponto de impacto (Pilkington \& Grieve 1992) e que o furo em questão situava-se a mais de $1000 \mathrm{~m}$ do centro geométrico da depressão (Fig. 8), a ausência de feições de metamorfismo de impacto nos fragmentos analisados não deve ser considerada como prova conclusiva da inexistência de cones de estilhaçamento ou de minerais de alta pressão, em pontos mais interiores da depressão.

A interpretação dos sismogramas das Figuras 4 à 7 permite estimar que a espessura do pacote sedimentar do interior da depressão entre $380-450 \mathrm{~m}$. A velocidade de $3300 \mathrm{~m} / \mathrm{s}$, relativamente baixa, calculada para o topo do embasamento pode ser explicada por um possível fraturamento deste, se assumirmos a sua origem por impacto meteorítico. Pela fórmula de Grieve \& Robertson (1979) para o cálculo da profundidade de crateras em alvos sedimentares, chega-se a espessuras de menos de $70 \mathrm{~m}$, nos levando a supor que a camada terciária, no momento do impacto era fina, de modo que o impacto pode ser considerado como tendo ocorrido diretamente no embasamento. Pela fórmula de Grieve \& Robertson (1979) para impactos em rochas cristalinas, a estimativa da profundidade da depressão de Colônia é de 435 m.

CONCLUSÕES O modelo de subsuperfície da depressão de Colônia, em sua porção centro-sul é mostrada na figura 8. A espessura do pacote sedimentar calculado a partir da interpretação dos sismogramas de campo e do modelamcnto direto cinemático situa-se entre 380 e $450 \mathrm{~m}$. A figura 9 apresenta as seções obtidas através da cratera a partir de diferentes métodos geofísicos. Nota-se que o método sísmico estima uma profundidade maior para a cratera. Entretanto, considerando-se as margens de erros de outros métodos, esta aparente discrepância se reduz drasticamente. A profundidade máxima de $450 \mathrm{~m}$ para o topo do embasamento cristalino é concordante com os valores obtidos a partir, não somente de considerações morfológicas por Riccomini et al. (1991), mas 


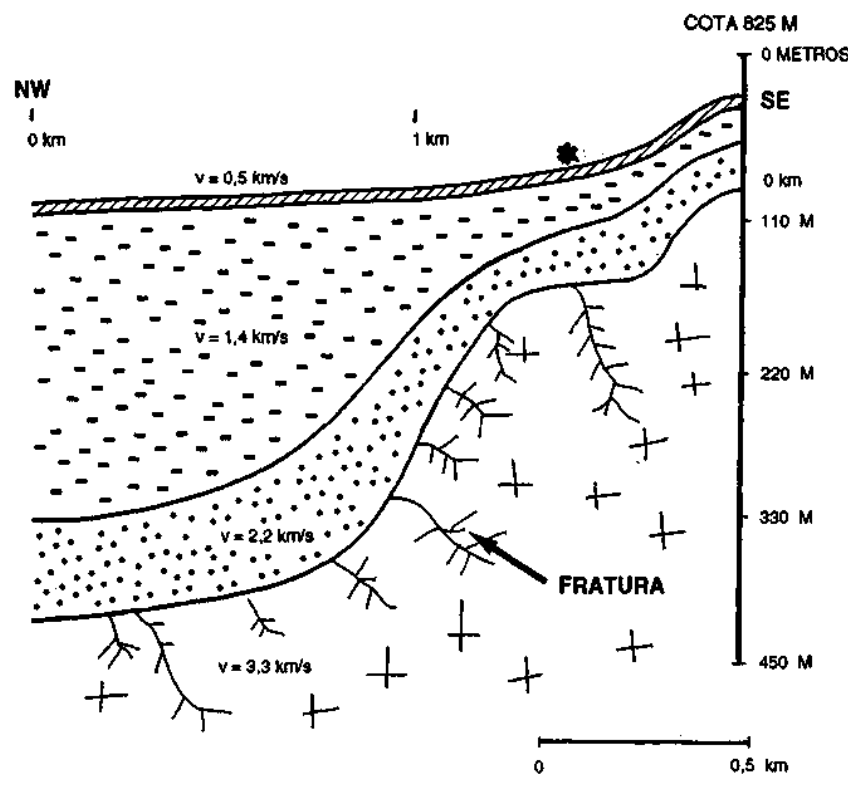

Figura 8. Seção geofisico-geológica SE-NW esquemática da parte centro-sul da depressão circular de Colônia. Exagero vertical 1:5. Notar a concordância morfológica entre o topo da camada de idade terciária $(2.2 \mathrm{Km} / \mathrm{s})$ e o topo do embasamento fraturado $(3.3 \mathrm{Km} / \mathrm{s})$. Também notar que a morfologia do topo da camada de idade quaternária $(1.4 \mathrm{Km} / \mathrm{s})$ é discordante com o topo da camada subjacente. A localização do poço perfurado pela Air Drill está indicada com um asterisco.

Figure 8. Schematic geophysical-geological SE-NW section of the central-south part of the circular depression of Colônia. Vertical exaggeration 1:5. Note the agreement in morphology between the top of the terciary layer $(2.2 \mathrm{Km} / \mathrm{s})$ and the top of the fractured basement $(3.3 \mathrm{Km} / \mathrm{s})$. Also, note the different morphology of the top of the quaternaty layer $(1.4 \mathrm{Km} / \mathrm{s})$ and the top of the underlying layer. The location of a well drillcd by Air Drill is indicated with an asterisc.

também pela fórmula empírica de Grieve \& Robertson (1979) e por dados magnetotelúricos por Massero \& Fontes (1992).

Baseando-se na concordância morfológica da camada terciária com o topo do embasamento, estimamos em aproximadamente 36 milhões de anos a idade máxima para a formação da depressão, como já estimado por Riccomini et al. (1991) e o obtido a partir da fórmula de Grieve \& Robertson (1979).

A baixa velocidade sísmica obtida para o topo do embasamento, pode ser devida à existência de brechas e/ou fraturas nesta unidade, talvez geradas pelo impacto. Alguns

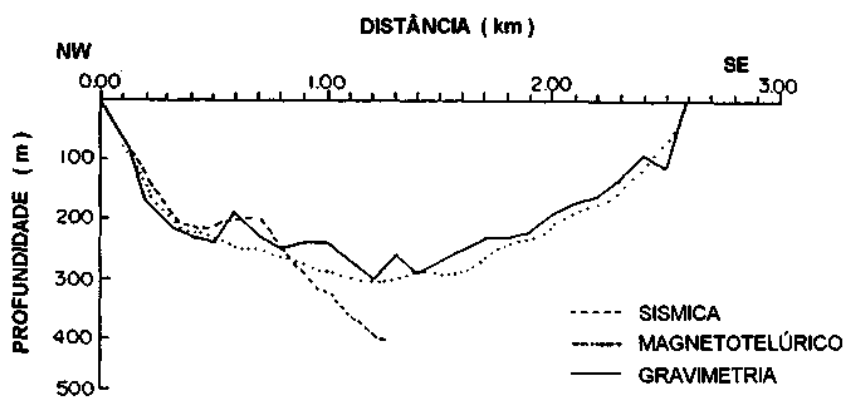

Figura 9. Seção NW-SE através da depressão circular de Colônia, obtida a partir de diferentes levantamentos geofisicos: sísmico (este trabalho), gravimétrico (Moita \& Flexor 1991) e magnetotelúrico (Massero \& Fontes 1992) Note a estimativa de uma maior profundidade para a cratera, obtida pelo método sísmico, mas dentro da margem de erro do método magnetotelúrico.

Figure 9. NW-SE section across the circular depression of Colônia, obtained different geophysical methods: seismic (this paper), gravimetric (Motta \& Flexor 1991) e magnetotclluric (Massero \& Fontes 1992). Note the estimate of agreaterdepth for the crater obtained by seismics, but within the error-bars of the magnctotelluric method.

sismogramas (linha A, Fig. 4), indicam a possível existência de falhas normais próximas à borda da cratera. Dados gravimétricos coincidentes com esta linha (Naomi Ussami, comunicação verbal) também indicam a presença desta estrutura.

As evidências sísmicas apresentadas neste trabalho reforçam a hipótese de impacto como origem da estrutura circular de Colônia. Contudo, a confirmação definitiva de uma origem por impacto meteorítico terá que ser baseada em evidências geológicas.

Agradecimentos O autor agradece ao pessoal do IAGUSP, especialmente, Luis Galhardo, José Roberto Barbosa e Célia Fernandes, pelo apoio em diversas etapas desta pesquisa; ao grupo de geofísica do IPT; bem como à Dra. Naomi Ussami pêlos dados de levantamento gravimétrico coincidente com uma das linhas sísmicas e, pelas críticas e sugestões durante a realização deste trabalho; ao Dr. Cláudio Riccomini, pelas discussões e ao Dr. Joel Valença, pela descrição petrográfica das lâminas delgadas e pela revisão do manuscrito; ao Dr. Marcelo Assumpção pela coordenação e incentivo, durante esta pesquisa, em todas as suas fases e à FAPESP pelo apoio financeiro.

\section{REFERENCIAS}

Almeida, F. F. M. 1964. Fundamentos Geológicos do relevo paulista. Boll. Inst. Geog. Geol., 41:167-262.

Cohen, J. K. \& Stockwell Jr., J. W. 1996. CWP/SU release 29, a free Seismic Software Environment for Unix Platforms. CWP-Colorado School of Mines.

Coutinho, J. M. V. 1980. Carta geológica da região metropolitana da grande São Paulo, 1:100.000, São Paulo, Emplasa, 2 folhas.

Crosta, A. P. 1982. Estruturas de Impacto no Brasil: uma síntese do conhecimento atual. In: CONGR. BRÁS. GEOL., 32. Salvador, 1982. Boletim de Resumos Expandidos... Salvador, SBG. v. 4, p. 1372-1377.

Dietz, R. S. 1961. Astroblemes. Scientific American. 205(2): 141-148.
Gandolfo, O. C. B. 1991. Levantamentos sísmicos de refração na depressão circular de Colônia. São Paulo, 105p. Trabalho de Graduação, Instituto Astronómico e Geofísico da Universidade de São Paulo).

Grieve, R. A. F. \& Robertson, P. B. 1979. The Terrestrial cratering record, I. Current status of observations. Icarus, 38:212-229.

Kollert, R.; Bjornberg, A. \& Davino, A. 1961. Estudos preliminares de uma depressão circular na região de Colônia: Sto.Amaro, São Paulo. Boi. Soe. Geol., 10:57-77.

Masero, W. C. B. \& Fontes, S. L. 1992. Geoeletrical Studies of The Colônia Impact Structure, Santo Amaro, State of São Paulo - Brazil. Rev. Brás. de Geof.. 10:25-41. 
Motta, U. S. \& Flexor, J. M. 1991. Estudo Gravimétrico da Depressão de Colônia, São Paulo-Brasil. In: Congresso Internacional da Sociedade Brasileira de Geofísica, 2. Salvador, 1991. Boletim de Resumos Expandidos ... Salvador, SBGf. v. 1, p. 140-142.

Pilkington, M. \& Grieve, R. A. F. 1992. The geophysical signature of terrestrial impact craters. Rev. of Gephysics., 30:161-181.

Riccomini, C.; Appi, C. J.; Freitas, E. L. \& Arai, M.; 1987. Tcctônica e Sedimentação no Sistema de Rifts Continentais da Serra do Mar (bacias de Volta Redonda, Resende, Taubaté e São Paulo). $1^{\circ}$ Simpósio de Geologia Rio de Janeiro-Espírito Santo, SBC. Anais ... v. I, 252-258.

Riccomini, C; Turcq, B.; Martin, L.; Moreira, M. Z.; Lorsheiter, M. L. 1991. The Colônia Astrobleme, Brazil. Rev. Insf. GeoL, 12:9-25.
Riccomini, C.; Neves, F. A. \& Turq, B. 1992. Astroblema de Colônia, São Paulo-Brasil: estágio atual de conhecimento. In: Congresso Brasileiro de Geologia, 37. São Paulo, 1992. Roteiro de Excursões, São Paulo, SBG. $14 \mathrm{p}$.

Sadowski, G. R. 1974. Tecíonica da Seira de Cubaíão, SP. São Paulo, 159p. (Tese de Doutoramento, Instituto de Geociências da Universidade de São Paulo).

Manuscrito A-912

Recebido em 13 de março de 1997

Revisão do autor em 03 de fevereiro de 1998

Revisão aceita em 05 de fevereiro de 1998 